ROCZNIKI HUMANISTYCZNE

Tom LXVIII, zeszyt 5 - 2020

DOI: http://dx.doi.org/10.18290/rh20685-4

\title{
POSTFIGURATIONEN DER SHOAH IM GEGENWÄRTIGEN FLÜCHTLINGSDISKURS ANHAND VON JENNY ERPENBECKS ROMAN GEHEN, GING, GEGANGEN (2015)
}

\begin{abstract}
A b s t r a k t. Jenny Erpenbecks Flüchtlingsroman erschließt durch die prozesshafte Konstruktion des Protagonisten und seine engagierte Begegnung mit den Flüchtlingen im gegenwärtigen Deutschland die grundlegende Komplementarität des Shoah-Narrativs und des aktuellen Asyl- und Flüchtlingsdiskurses in Europa. Die vom pensionierten Professor für Altphilologie internalisierte Anklage des Verfalls der Menschenrechte verleitet ihn dazu, in den Referenzen zur Shoah und zum Kolonialismus ,Zuflucht` zu suchen. Das Paradoxe dieser Transfiguration besteht darin, dass die Unsagbarkeit des Judenmordes im Roman dazu genutzt wird, die gesellschaftliche Gegenwart $\mathrm{zu}$ beleuchten und die Pervertierung der europäischen Gesetzgebung, welche auf die Shoah als Initialzündung der modernen Menschenrechte gründet, anzuklagen.
\end{abstract}

Schlüsselwörter: Migrationsroman; Transfiguration; Shoah.

Die aktuelle, seit einigen Jahren andauernde Flüchtlingskrise in Europa entzündet sich an den immer wieder zurückkehrenden Brennpunkten, für die bislang keine Lösungen in Sicht sind. Proteste der nach Europa vor Krieg und Terror Geflüchteten richten sich in erster Linie gegen das europäische Asylrecht, das durch das sog. Dublin II-Abkommen sanktioniert wurde, und ihnen Arbeitssuche und Freizügigkeit verbietet. Die prekäre Lage der Flüchtlinge verschärfen darüber hinaus überfüllte Asylheime und chronisch überforderte Behörden, die das Asylverfahren übermäßig lang hinauszögern. Parallel dazu spielen sich $\mathrm{zu}$ derselben Zeit außerhalb Europas existentielle Notsituationen ab, dramatische Meeresüberfahrten, bei denen täglich Menschen ums Leben kommen oder der Willkür der skrupellosen Schlepper aus-

Prof. Dr. habil. ANNA RUTKA - Katholische Universität Johannes Pauls II. in Lublin, Institut für Literaturwissenschaft, Lehrstuhl für deutsche und deutschsprachige Literatur des 20. und 21. Jahrhunderts; Korrespondenzadresse: Aleje Racławickie 14, 20-950 Lublin; E-Mail: wiosna@kul.pl. ORCID: https://orcid.org/0000-0002-0872-8149. 
gesetzt sind. Aus der innereuropäischen Perspektive scheinen nativistische und nationalstaatliche Diskussionen über Sicherheit, Terrorismus, finanzielle Überforderung der Sozialkasse bis hin zur xenophoben Haltung und den Angst schürenden Protestrufen gegen sog. kulturelle ,Überfremdung ${ }^{61}$ Europas kein Ende zu nehmen. Der tagespolitische Diskurs transkultureller Migration, der im öffentlichen Raum ganz offensichtlich als Krise und Prekarisierungsprozess verhandelt wird, hält aktuell Einzug in das Medium der Literatur. Literarische Erzähltexte repräsentieren und reflektieren dabei gesellschaftliche Diskurse und stellen gleichzeitig einen Teil dieser Strukturen dar, indem sie diskursive Mechanismen im Umfeld der sog. europäischen Flüchtlingskrise normieren und produzieren.

Von den Prosawerken, die die gegenwärtigen Probleme der Flüchtlinge und Asylsuchenden wie auch der globalen Migrationsbewegung nach Europa aufgreifen, möchte ich z. B. auf den bereits 2008 von Julya Rabinowich verfassten Roman Die Erdfresserin verweisen. Die Erdfresserin erzählt die Geschichte einer aus Dagestan nach Westeuropa emigrierten Frau, die nach dem Zerfall der Sowjetunion von einer katastrophalen ökonomischen Situation ihres Landes und einer gleichzeitigen familiären Misere zur Arbeitsmigration gezwungen wurde. Auf die Flüchtlingskrise in Europa geht explizit Elfriede Jelinek in ihrem Collagetext Die Schutzbefohlenen (2013) ein, in dem die Asylproteste um die Wiener Votivkirche mit Aischylos' Drama Die Schutzflehenden und der Kritik neoliberaler Machtverhältnisse im Europa des 21 . Jh. verwebt werden. ${ }^{2} 2012$ erschien der Debütroman des österreichischen Autors Martin Horváth Mohr im Hemd. Oder wie ich auszog, die Welt $z u$ retten. Der an die pikareske Tradition des Schelmenromans angelehnte Prosatext Horváths spielt in einem Wiener Asylbewerberheim für minderjährige, unbegleitete Flüchtlinge und gewährt aus einer komödiantischsubversiven Froschperspektive eines fünfzehnjährigen afrikanischen Jungen ,schelmische' Einblicke in die Schicksale und die ausweglosen Existenzlagen der jugendlichen Flüchtlinge in Europa. ${ }^{3}$ Erwähnenswert in diesem Zu-

\footnotetext{
${ }^{1}$ Vgl. Thilo Sarrazins Streitschrift Deutschland schafft sich ab. DVA, 2010.

${ }^{2}$ Jelinek knüpft in ihrem postavandgardistischen Kunsttext direkt an den Hungersprotest von etwa 60 jungen Männern aus Pakistan, Nordafrika und Afghanistan in Wien in den Jahren $2012 / 13$ an.

${ }^{3}$ Eine ausführliche Analyse des Romans liefert die Dissertation von Daniela H. Roth: Migration und Adoleszenz: Die (Un-)Möglichkeit transnationaler Handlungsfreiheit in Alina Bronskys ,Scherbenpark' (2008), Steven Uhlys ,Adams Fuge“ (2011) und Martin Horváths, Mohr im Hemd oder wie ich auszog, die Welt zu retten' (2012). madoc.bib.uni-mannheim.de/44302/1/Roth\%20 Daniela_Die\%20Unm\%C3\%B6glichkeit\%20transnationaler\%20Handlungsfreiheit.pdf. 4 June 2018.
} 
sammenhang wären auch Prosatexte neueren Datums wie etwa Abbas Khiders Roman Ohrfeige (2016) über einen irakischen Flüchtling, der mit einer fingierten Lebensgeschichte die beim Asylverfahren entscheidenden Richter beeindrucken und überzeugen möchte. Ein ästhetisch beachtenswertes Werk lieferte 2017 Ilija Trojanow. In einer aus reflexivartigen Episoden kunstvoll komponierten Notatensammlung erstellt der Autor eine parabolische und zugleich sehr poetische „Topographie des Lebens nach der Flucht“ (Trojanow, Klappentext), indem er den Erlebnishorizont der Geflüchteten des 21. Jh. exemplarisch-metaphorisch verdichtet.

2015 veröffentlichte Jenny Erpenbeck ihren Roman Gehen, ging, gegangen, der von der Kritik unisono als der aktuelle Flüchtlingsroman apostrophiert wurde. Das Werk erschien genau zu dem Zeitpunkt, als die Migration von Hunderttausenden vor Krieg und Terror Geflüchteten ein in der neueren Geschichte Europas unbekanntes Ausmaß annahm. Die Kritiker attestierten Jenny Erpenbeck ein „[s]eismographisches Gespür“ (Bremerich), da sie mit ihrem sensiblen Einblick in die existenziellen Notlagen und Herkunftsgeschichten der afrikanischen, in Berlin gestrandeten Flüchtlinge vieles vorweggenommen hatte, was erst nach 2015 ins öffentliche und private Bewusstsein Einzug fand.

Die Autorin präsentiert die Geschichte von einer Gruppe junger nordafrikanischer Männer, die auf dem Berliner Oranienplatz in einen Hungerstreik treten, aus der Perspektive des pensionierten Altphilologen Richard. Durch Zufall war der lebensferne Professor auf die Situation der protestierenden Flüchtlinge aufmerksam geworden. Mit der Zeit lernt der anfangs distanziert-gleichgültige Protagonist die individuellen Schicksale der Männer kennen, besucht sie in ihren Unterkünften, engagiert sich für ihre Asylverfahren und nimmt zum Schluss einige von ihnen angesichts ihrer ausweglosen Lage bei sich zu Hause auf.

Gehen, ging, gegangen erschließt den Leserinnen und Lesern die prekäre Welt der Asylsuchenden, die, „nachdem sie die Überfahrt über ein wirkliches Meer überlebt haben, nun in Flüssen von Akten ertrinken“ (Erpenbeck 310). Neben dieser tagespolitisch brisanten Dimension des Romans ${ }^{4}$ erscheint der innere Bewusstseinsprozess des Protagonisten als sehr aufschlussreich und qualifiziert den Text gattungsmäßig als eine Art aktuellen

\footnotetext{
${ }^{4}$ Vgl. die Kommentare der Kritik: „Roman der Stunde” (Frankfurter Rundschau vom 18.09. 2015), „Roman der Saison” (Süddeutsche Zeitung vom 31.08.2015), ,sorgfältig recherchierter Tatsachenroman“ (Frankfurter Allgemeine Zeitung vom 27.08.2015), „der Roman zur politischen Situation“" (Die Welt vom 29.08.2015)
} 
,Entwicklungsroman'. Die literarische Konstruktion der Hauptfigur als emeritierter Universitätsprofessor für Altphilologie, der in vielerlei Hinsicht selbst ein „Gestrandeter“ (Bremerich) ist, da er Nachkriegszeit, Entstehung und Fall der DDR und den Systemwechsel erlebt hatte, macht ihn zu einem paradigmatischen Beispiel eines deutschen Bildungsbürgers, dessen Vita die zentralen Eckpfeiler des mitteleuropäischen Gedächtnisses widerspiegelt. Seine klassische Ausbildung kombiniert mit den Lebenserfahrungen, die die bedeutendsten historischen Umbruchereignisse des 20. und 21. Jh. kondensieren, machen ihn $\mathrm{zu}$ einer Demonstrationsfigur „multidirektionalen Gedächtnisses" (Rothberg 37-63), das allerdings im Text nicht als Ist-Zustand dargelegt wird, sondern als ein mühsamer, schrittweiser Vorgang einer postmodernen und poststrukturellen Erkenntnisbewegung konzipiert wird. Bei jenem „Selbstbespiegelungsprozess“"5, der gleichzeitig eine kritische Hinterfragung der axiologischen Kondition der gegenwärtigen europäischen Gesellschaft ist, geht der Protagonist und Ich-Erzähler reflexiv vor, indem er bei der Deutung von zeitgenössischen Ereignissen figurative und motivische Referenzen zu antiker Kultur, europäischer Kolonialgeschichte, dem Systemwandel in Deutschland und nicht zuletzt zur Zeit des Nationalsozialismus und zur Shoah erstellt. Die Analyse der Flüchtlingskrise, die in den Gesprächen Richards mit den Flüchtlingen dargeboten wird, geht einher mit frustrierenden Erkenntnissen und einer desillusionierenden Dekonstruktion aufklärerischer Tradition und den demokratischen Ideen des gegenwärtigen Europas. Ausschlaggebend erscheint bei den Reflexionen des Protagonisten der postfigurative Bezug auf Bilder und Zeichen der europäischen Judenvernichtung und der Kolonialisierungsgeschichte, die als Berührungsassoziationen im Sinne einer Kontiguität der metonymischen Strategie ${ }^{6}$ in den Erzählfluss aufgenommen werden. Jene metonymische Berührung als narrative Herstellung einer Beziehung zwischen disparaten Sachbereichen oder Phänomenen dient, wie es der skandinavische Dramatiker Jon Fosse bemerkt, dazu, „Zuflucht $[\mathrm{zu}]$ nehmen“, „[...] zu dem, was vorhanden ist, wozu man Zuflucht nehmen kann und nicht zu dem, was bloß ähnlich ist. Man benutzt also eine

\footnotetext{
${ }^{5}$ Sibylle Birrer bemängelt bei der Konstruktion der Hauptfigur, dass sie nie über „Selbstbespiegelung" eines satten Westlers hinausgeht (Birrer).

${ }^{6}$ Roman Jakobson unternimmt in seinem Beitrag Randbemerkungen zur Prosa des Dichters Pasternak (1935) eine wichtige Unterscheidung zwischen der Ähnlichkeitsassoziation der Metapher und der Berührungsassoziation der Metonymie. Während eine Metapher von der Similarität zweier oder mehrerer Bilder ausgeht, stellt das Umbenennen bzw. Neubenennen des Metonymischen eine Berührungsbeziehung dar als Ersetzung des eigentlich Gemeinten durch etwas anderes, das mit ihm real-sachlich oder geistig korrespondiert. (Jakobson 357-374).
} 
sprachliche Trope, um zu sagen, was man nicht sagen kann, was man trotzdem gesagt haben muß“ (Kjaerstad und Fosse 18). Im gedächtnistheoretischen Sinne kann man den Transfigurationsvorgang mit dem von Leslie A. Adelson vorgeschlagenen Konzept der „touching tales“ in eine aufschlussreiche Verbindung bringen. Mit „touching tales“ meint Adelson erstmals im Kontext des deutsch-türkischen Verhältnisses in der Literatur der 1990er Jahre eine mögliche Strategie zur narrativen Verarbeitung europäischer Vergangenheit:

[B]road historical narratives of barbarism and civilization are often the (teleo)logical touchstones on which evaluative accounts of the Third Reich and its place in modernity rely, as do those of the so-called Islamic "Orient" and its place in Europe. In this broad narratological sense twentieth-century tales of Germans and Jews are not so much analogous to those of Germans and Turks as they are proximate. In a word, they "touch." (Adelson 98)

Texte, in denen deutsche, türkische und jüdische Figuren und ihre narrativen Vergangenheitsdiskurse aufeinandertreffen, sind nach Adelson Berührungsgeschichten, ,because they evoke a culturally residual, referentially nonspecific sense of guilt, blame, shame, and danger" (Adelson 102).

In Erpenbecks Roman berühren und kreuzen sich auf der Folie der Gesellschaftsgegenwart des Protagonisten Lebens- und Erinnerungsräume unterschiedlicher Couleur. Die deutsche Geschichte und aktuelle Flüchtlingspolitik werden in ein Netz pluraler Perspektiven und Vergangenheitsnarrationen eingebettet. Einen der wichtigen Referenzpunkte innerhalb der Narration stellen die Shoah-Postfigurationen dar. Die Schicksalsschläge der afrikanischen Männer, ihre rechtlich und existenziell subalterne, prekäre Situation als Personen, die aus Zeit und Raum „herausgefallen“ (Erpenbeck 51) sind, werden an einigen Stellen metonymisch mit der Reflexion über jüdische Opfer des nationalsozialistischen Staates in Adelsons Sinne als aufeinander folgende (proximale), sich berührende Geschichten kurzgeschlossen. Die Postfigurationen der Shoah werden in Richards Überlegungen äußerst dezent als Randbemerkungen eingeschoben. Der Protagonist und Ich-Erzähler zugleich geht mit den postfigurativen Strategien sehr behutsam um und vermeidet jegliche Gleichsetzung im Sinne der Similarität.

Einen signifikanten Ausgangspunkt für postfigurative Kontexteinschreibung und aktualisierende Überschreibung der Holocaustmotive bildet der Fernsehbericht über die hungerstreikenden Asylsuchenden auf dem Berliner Alexanderplatz, bei dem Richard erstmals seine Gleichgültigkeit und die 
Austauschbarkeit der Bilder bewusst werden (Erpenbeck 27, 29). Demzufolge stellt er sich die Frage nach der Erzählung, die „den beliebigen Bildern heutzutage zugrunde“ (Erpenbeck 29) liegt. Die in den Text eingeschobenen transfigurativen Reflexionen der Kolonial-, Nationalsozialismus-, Wendeund Shoah-Geschichte fungieren folgerichtig als Versuche, ,Zuflucht zu finden' und als Wege zum Kenntlichmachen dessen, was gesagt werden muss, und aus irgendwelchen Gründen nicht gesagt werden kann. Bevor ich auf die Inhalte eingehe, die gegenwärtig im europäischen Flüchtlingsdiskurs medial und politisch als Krise verhandelt werden, möchte ich auf einige exemplarische Romanstellen hinweisen, an denen die Berührungsreferenzen zum jüdischen Schicksal strategisch hergestellt werden.

Die Begegnungen und Gespräche mit afrikanischen Geflüchteten aus dem Asylheim, die Richard jeden Tag besucht, führen bei ihm zu einem signifikanten Einbruch der euroatlantischen, hegemonialen Gedächtnisperspektive: „Die Afrikaner wussten bestimmt nicht, wer Hitler war“ (Erpenbeck 64). Eine ähnliche Diskrepanz im Wissens- und Bewusstseinshorizont konstatiert er auch in Bezug auf andere gedächtnisstiftende Ereignisse wie etwa die Berliner Mauer, den Kalten Krieg, die Teilung Deutschlands. Darüber hinaus hält es der Protagonist für unmöglich, den afrikanischen Flüchtlingen die Geschichte des „fabrikmäßige[n] Ermorden[s] von Menschen“ (Erpenbeck 150) zu vermitteln: „Er schämt sich dafür plötzlich so sehr, als sei das, was jeder hier in Europa weiß, sein ganz persönliches, niemandem auf der Welt zumutbares Geheimnis“ (Erpenbeck 150). Der Topos der Unaussprechbarkeit der Shoah wird somit oberflächlich aufrechterhalten. Dennoch greift Richard manchmal latent und manchmal ganz offen zu den Shoah-Vorstellungen, um sozusagen stellvertretend über die schmerzliche Gegenwart zu sprechen; Die Asylsuchenden sind auf ein aussichtsloses Warten angewiesen, was sie quasi zu Gefangenen in einem zeitlichen Entweder-Oder verdammt. Im sicheren Europa fallen die Asylsuchenden nicht nur aus dem elementaren Zeit-RaumRahmen heraus, sondern auch aus den soziokulturellen Koordinaten des Gesellschaftslebens. Ihnen werden nämlich Rechte auf Arbeit, Bewegungsfreiheit und Bestimmungsrecht über ihre Körper und Zukunft verweigert.

Der Roman legt eine tiefgründige Kluft bloß. Während Richards Zeitverständnis „eine Reihe von disziplinierten, immer gleich aussehenden Tätigkeiten“ (Pokrywka 415) umfasst, was übrigens als Verwirklichung eines traditionsreichen bürgerlichen Lebensideals gilt, erscheinen ihm die Flüchtlinge als „Tote auf Urlaub“, Menschen, die wie Opfer der Nationalsozialisten metaphorisch und wirklich in einer Übergangszone zwischen Leben und Tod 
suspendiert bleiben. ${ }^{7}$ Richard vertieft diesen auf die letzten Worte des kommunistischen Revolutionärs Eugene Levine zurückgehenden Gedanken folgendermaßen: „Den Unterschied zwischen den Flüchtlingen, die heutzutage auf dem Meer irgendwo zwischen Afrika und Europa ertrinken, und denen, die nicht ertrinken, macht allein der Zufall. In diesem Sinne ist auch jeder von den afrikanischen Flüchtlingen hier, denkt Richard, ein Lebendiger und ein Toter" (Erpenbeck 2015: 208). Die in Bezug auf die jüdischen Opfer literarisch tradierte Transposition als lebendige Tote ${ }^{8}$ bildet den zentralen Punkt der metonymischen Berührungsassoziation im Roman im Hinblick auf Europas Flüchtlinge des 21. Jahrhunderts. Die aufgrund des Dublin IIAbkommens konstituierten Asylgesetze sprechen den Geflüchteten alle elementaren Grundrechte (Arbeit, Freizügigkeit, Wohnung etc.) ab, stigmatisieren sie im staatlich-bürgerlichen Sinne als „weniger schützenswert“ (Lorey 2012: 37) und heben somit „die Trennlinie zwischen Geistern und Menschen“ (Lorey 2012: 274) auf. Die Erlebniswelt der nach dramatischer Meeresüberfahrt nur per Zufall am Leben Gebliebenen wird im Gestus der rhetorischen Umkehrung der vom Ich-Erzähler in die Gegenwart transportierten Bilder den Shoah-Opfern nahe gebracht:

Schon oft hat er gedacht, dass alle Männer, die er hier kennengelernt hat, genauso gut am Grund des Mittelmeers liegen könnten. Und umgekehrt, dass all diejenigen Deutschen, die während des sogenannten Dritten Reiches umgebracht wurden, Deutschland als Geister noch immer bewohnen, all die Fehlenden und auch deren ungeborene Kinder und Kindeskinder gehen, denkt Richard manchmal, neben ihm auf der Straße, sind unterwegs zur Arbeit oder zu Freunden, sitzen unsichtbar in den Cafés, spazieren, kaufen ein, besuchen Parks und Theater. Gehen, ging, gegangen. Die Trennlinie zwischen Geistern und Menschen war für ihn, und er weiß nicht, woran das liegt, schon immer sehr dünn, mag sein, weil er selbst damals, als Säugling, in den Wirren des Krieges so leicht hätte verlorengehen und ins Totenreich abrutschen können. (Erpenbeck 2015: 247, 248).

\footnotetext{
${ }^{7}$ An dieser Stelle erscheint als Referenz der berühmte Roman von Milo Dor unter ebendiesem Titel Tote auf Urlaub aus dem Jahre 1952.

${ }^{8}$ In der Literatur nach Auschwitz ist die Utopie der Wiederauferstandenen eine oft benutzte Trope. Sie zielt laut Bastian Reinert darauf, dem Massenmord etwas entgegenzusetzen. Das Motiv der lebendigen Toten wurde literarisch in Bezug auf den Auschwitz-Diskurs von Elfriede Jelinek (z. B. In den Alpen, Erlkönigin, Die Kinder der Toten) oder von George Tabori (Das Jubiläum) eingesetzt (Reinert 259-273). In diesem Kontext ist es auch wichtig, auf Theodor Adornos Negative Dialektik zu verweisen. Der Überlebende hat nach Adorno sein Überleben mit den ihn immer wieder heimsuchenden traumatischen Träumen zu bezahlen, „daß er gar nicht mehr lebte, sondern 1944 vergast worden wäre, und seine ganze Existenz danach lediglich in der Einbildung führte“" (Adorno 354).
} 
Die metonymische Berührung der afrikanischen Flüchtlinge und der Holocaust-Opfer in ihrem Status als „lebendige Tote“ bzw. Geister und Träger des Gedächtnisses erhält hier den Status eines Korrektivs der Gegenwart. Sie verweist nämlich auf die Relevanz des Menschenrechtsdiskurses und auf dessen Pervertierung in der öffentlichen Realität Deutschlands und Europas. Erpenbecks Roman gewährt Einblicke in die Konsequenzen der europäischen Asylgesetzregelungen für einzelne individuelle Schicksale. Als Folge werden Ghettoisierung und ausschließende Diskriminierung deutlich: Richards afrikanische Gesprächspartner absolvieren einen langen bürokratischen Weg durch diverse Instanzen und Behörden. Die „erkenntnisdienstliche[n] Behandlung[en]" (Erpenbeck 249) haben eigentlich nur ein einziges Ziel, sie nach Italien (zum Erstaufnahmestaat) abzuschieben und ihnen zu beweisen, dass sie nicht berechtigt sind, in Deutschland eine selbstständige Existenz aufzubauen. Arbeitsrecht und sogar Ortswechsel innerhalb Deutschlands stehen ihnen selbstredend nicht zu (Erpenbeck 257). Die im nicht relativierbaren Menschenrechtsdiskurs konstitutive „Frage nach der Macht über das eigene Leben“, bleibt, so Richards Fazit, immer noch ,in erster Linie eine Frage der Macht und nicht die nach dem Leben“ (Erpenbeck 272). Das Verdienst von Erpenbecks Literarisierung ist, den Stimmlosen, weil lebendig Toten, durch die transfigurative Zusammenstellung mit den lebendigen Toten des Holocaust, eine hörbare Stimme zu verleihen, die dem öffentlich Verdrängten oder Vergessenen sein Gegenteil vorhält. Die Verdeutlichung dieser Strategie erfolgt auch mithilfe der Verschränkung der innereuropäischen (hegemonialen) Perspektive des emeritierten Professors mit der existenziellen Prekarisierung der Flüchtlinge. Richard macht einen Umdenkprozess durch. Als Altphilologe verfügt er über ein fundiertes Wissen über die Grundlagen des Menschenrechtdiskurses: das Römische Recht, antike Ideen der Gleichheit aller Menschen bei Seneca, Ovid und Plato bis hin zu Aufklärungsdiskursen Kants, Hobbes', Lockes und Rousseaus. Als deutscher und europäischer Bürger steht er selbstverständlich für die Einhaltung der Menschenrechte ein. Dennoch muss er im Gespräch mit dem nach Italien abzuschiebenden Libyer Awad entrüstet konstatieren, dass seine aufgeklärte, europäische Bürgerlichkeit angesichts der elementaren Überlebensprobleme der heutigen Migranten verstummen muss: „Aber Richard, was soll man essen? [wenn man nach Italien ohne Arbeitsrecht abgeschoben wird, A.R.] Richard hat Foucault gelesen und Baudrillard und auch Hegel und Nietzsche. Aber was man essen soll, wenn man kein Geld hat, um sich Essen zu kaufen, weiß er auch nicht“" (Erpenbeck 81). 
Die Pervertierung des Menschenrechtsdiskurses in der aktuellen europäischen Asylgesetzgebung, Abschiebe- und Duldungsregelung drückt der Protagonist in folgender Reflexion aus: „Das Praktische an einem Gesetz ist, dass niemand es persönlich gemacht hat und daher niemand persönlich dafür zuständig ist“" (Erpenbeck 270). Beim Lesen der offiziellen Kundgebung der Asylbehörde, die an die Afrikaner gerichtet ist, leuchtet Richard ihre Vernunft instrumentalisierende Macht ein. Er hält fest: „Das Gesetz verlagert sich tatsächlich von der physischen Wirklichkeit ins Reich der Sprache.“ (Erpenbeck 86). Die Asylgesetze, die der Protagonist seinen afrikanischen Schützlingen mit wenig Erfolg verständlich zu machen versucht, erwecken eine markante Berührungsassoziation zu den juristisch-ideologischen Grundlagen des Dritten Reiches. Die von Horkheimer und Adorno in ihrer Dialektik der Aufklärung erschlossene Erkenntnis über die Degradierung des kantschen utopischen Weltentwurfs vom Ausgang der Menschheit aus der selbstverschuldeten Unmündigkeit zum kalkulierenden Denken der reinen Vernunft findet in Erpenbecks Flüchtlingsgeschichte eine erschütternde Aktualisierung. Die afrikanischen Geflüchteten machen die Erfahrung, dass europäische Gesetze die Menschen ,präparieren' und klassifizieren. Sie erscheinen dem Protagonisten, der die Asylsuchenden in ihrem Status als Individuen kennengelernt hatte, als „[...] hochentwickelte, reine Gesetze, so sauber formuliert, dass die gefühlsmäßige Grundlage überflüssig geworden und praktisch nicht mehr existent ist" (Erpenbeck 309). Vernunft ist, um mit Horkheimer und Adorno zu sprechen, zur „Handhabe der Unterdrückung anderer" (Adorno 134) verkommen.

Den Höhepunkt des scheinheiligen Menschenrechtsdiskurses und der verkommenen Schutzpflicht des Staates stellen im Roman Szenen der Räumung des Asylbewerberheims und der Erstellung von Namenslisten der zu deportierenden Flüchtlinge dar. Die Szenerie und Durchführung der Räumungsaktion sowie diskriminierende und brutale Vorgehensweisen der Polizisten evozieren Stimmungen, die das Erzählte unweigerlich in die Nähe zum Faschismus rücken. Die offizielle Ausländerbehörden-Sprache, in der wortwörtlich von Räumung, Abtransport und Listen (Erpenbeck 257, 259) die Rede ist, stellen Transfigurationen des Shoah-Diskurses dar; ebenso wie die darauffolgende Beschreibung des Polizeieinsatzes aus der Perspektive des beobachtenden Protagonisten transfigurativ Bilder der Vergangenheit hervorruft: 
Jetzt entsteigen den vorderen Mannschaftswagen Polizisten in voller Montur: Kampfanzüge, Helme mit heruntergekapptem Visier, Knüppel, Pistole. Gewaltig dröhnt die Erde unter ihren Füssen, als sie marschieren. In Viererreihen nehmen sie Aufstellung vor dem Tor zum Asylbewerberheim. Richard fragt sich, ob tatsächlich 40 schwerbewaffnete Männer notwendig sind, um 12 afrikanische Flüchtlinge aus so einem Heim zu tragen, ganz zu schweigen von den übrigen rund 150 Polizisten, die in den anderen Wagen auf ihr Startsignal warten. Morgen, das weiß er jetzt schon, wird in der Zeitung stehen, wieviel der Einsatz gekostet hat, und die Kosten werden vom Volk der Buchhalter den Objekten des Abtransports als Schuld zugeschrieben werden, wie das auch in anderen Zeiten, wenn Deutschland irgendwen hat abtransportieren lassen, üblich gewesen ist. (Erpenbeck 258, 259)

\section{FAZIT}

Als die Allgemeine Erklärung der Menschenrechte im Jahre 1948 von den Vereinten Nationen unterzeichnet wurde, erwies sich die Erinnerung an den Holocaust als eines der wichtigsten Faktoren bei der Konstituierung des Menschenrechtskonzeptes. Heutzutage hat sich, wie dies Daniel Levy und Natan Szneider in ihrem vielbeachteten Buch Erinnerung im globalen Zeitalter. Der Holocaust (Erstausgabe 2001) überzeugend ausgelegt haben, das kosmopolitische Verständnis des Holocausts weitgehend etabliert. Holocaust als Modus der kosmopolitischen Erinnerung bestimmt „die Parameter entorteter Erinnerungslandschaften in der Zweiten Moderne, wird zu einem Modell der nationalen Selbstkritik, dient der Verbreitung von Menschenrechten als legitimatorischem Prinzip der Weltgesellschaft und wirkt als negative Erinnerung an den Umgang mit Alterität schlechthin“ (Levy und Szneider 238).

Jenny Erpenbecks Flüchtlingsroman erschließt durch die prozesshafte Konstruktion des Protagonisten und seine engagierte Begegnung mit den Flüchtlingen im gegenwärtigen Deutschland die grundlegende Komplementarität des Shoah-Narrativs und des aktuellen Asyl- und Flüchtlingsdiskurses in Europa. Die Schriftstellerin gestaltet ihren Text vorsätzlich als Politikum ${ }^{9}$, indem sie den Verrat am Menschenrechtsgedanken in der westlichen Gesellschaft deutlich macht. Den Kernpunkt von Richards Umdenken bildet die Bewusstmachung eben dieses aktuellen Eklats. Das Fazit nach der Lektüre von Gehen, ging, gegangen könnte deshalb lauten, dass das, was laut der

\footnotetext{
${ }^{9}$ Gerade diese Intentionalität des Romans wurde von einigen Kritikern stark kritisiert und als ästhetische Schwäche bemängelt. Vgl. z.B. (Buchzig; Lühmann).
} 
Fosseschen Definition der transfigurativen Strategie, nicht gesagt werden kann, aber was man gesagt haben muss, der klägliche Verfall des auf Aufklärungsidealen und Holocaust-Erinnerung gründenden Menschenrechtsgedanken ist. Diese vom pensionierten Professor für Altphilologie internalisierte Anklage verleitet ihn dazu, in den Referenzen zur europäischen Judenvernichtung und zum Kolonialismus narrative Zuflucht zu suchen. Das Paradoxe dieser Transfiguration besteht darin, dass die Unsagbarkeit des Judenmordes ${ }^{10}$ im Roman dazu genutzt wird, um gesellschaftliche Gegenwart zu beleuchten und die Pervertierung der europäischen Gesetzgebung, welche auf die Shoah als Initialzündung der modernen Menschenrechte gründet, anzuklagen. Erpenbecks Botschaft scheint in dieser Hinsicht unmissverständlich klar zu sein: Mitten im demokratischen, freien und globalisierten Europa werden die Menschenwürde und die Freiheit zu Leerformeln ausgehöhlt. Die persönlich erlebbar gemachten Flüchtlingsschicksale, für die sich der emeritierte Altphilologe einsetzt, bis zum verzweifelten Akt der provisorischen Unterbringung der Afrikaner bei sich zu Hause, lassen den Shoah-Diskurs aktuell neu aussprechbar und kulturell-gesellschaftlich effizient erscheinen. Auf der anderen Seite kann diese transfigurierende Literarisierung wohl begründete Vorbehalte gegenüber der Instrumentalisierung des realen Geschehens des Judengenozids für aktuelle geschichts- und politisch-didaktische Zwecke auslösen. Die Kritiker und Interpreten des Romans haben den offensichtlichen Rückgriff Erpenbecks auf nichtfiktionale Diskurse (Pokrywka $416,417)$ und eine Überschreitung der „Grenze zur außerliterarischen Realität" (Hermes 181) bemängelt und auf die dadurch herbeigeführte Minderung des literarischen Anspruchs des Textes hingewiesen. Obwohl Erpenbecks Arbeit die Berührungsassoziationen zu der Shoah mit großer Umsicht einsetzt und an keiner Stelle dem Versuch einer Similarität das Wort zuspricht, ist der Vorwurf, das Verbrechen an den europäischen Juden zum Objekt aktueller Explikationen zu machen, nicht von der Hand zu weisen, zumal die Transpositionen der Shoah an vielen, oben analysierten Stellen den Eindruck einer ,gesteuerte[n] Konkretisierung der dargestellten Welt“ (Pokrywka 417) erwecken mögen. Durch die Referenzen auf die Verbrechen des Dritten Reiches und seine gedanklichen Grundlagen werden die narrativen Schlüssel-

\footnotetext{
${ }^{10}$ Die ästhetischen und ethischen Kontroversen um die Fragen der Darstellbarkeit des Judenmordes waren und sind bis heute virulent. Als ausschlaggebend gelten in dieser Hinsicht die berühmte Äußerung von Theodor Adorno (Nach Auschwitz ein Gedicht zu schreiben, ist barbarisch) (Laermann 11-15), oder Elie Wiesel (,A novel about Treblinka i seither not a novel or not about Treblinka“) (Wiesel 7).
} 
stellen des Romans gleichsam per se ausgelegt, was die genuin literarischen Spielräume der metaphorischen Darstellung sichtlich einschränkt. Diese ästhetisch-narrative ,Falle' führt uns erneut zu der grundlegenden Frage, ob und inwieweit der Mord an europäischen Juden in Erzählmuster und ästhetische Strategien gebracht werden darf.

So plausibel die Vorwürfe und Bedenken gegenüber Erpenbecks offensiver Narrativierung und den daraus resultierenden literarischen Schwächen auch sein mögen, der ethische Wert der Aktualisierung der Shoah im Kontext der akuten europäischen Migrationsdebatten scheint davon nicht betroffen zu sein. Eine der zentralen Metaphern des Romans, die titelgebende Tempusformel - gehen, ging, gegangen -, artikuliert die Reflexion über die lineare, fortschreitende Zeitauffassung und impliziert zugleich Fortschrittsoptimismus und Bewegungsfreiheit eines jeden modernen Menschen. Richards ,Flüchtlingsabenteuer" mündet jedoch in eine pessimistische Erkenntnis, die sich auf Europas Migranten bezieht: „Die Zeit, in der ein Mensch nicht weiß, wie sein Leben ein Leben werden kann, füllt so einen Untätigen vom Kopf bis zu den Zehen.“ (Erpenbeck 293). Zu der schmerzlichen Desavouierung der Zeit-Kategorie gesellt sich im Falle der nach Europa Migrierten der Verfall der Bewegungsfreiheit. Was den Europäern wie Richard wie selbstverständlich zusteht, wird den anderen ebenso selbstverständlich abgesprochen. Allen Männern aus dem Asylheim wird zum Schluss das Aufenthaltsrecht in Deutschland verweigert. Die Abschiebung nach Italien steht ihnen bevor. Auf diese Aporie bezieht sich die auf zwei leeren Seiten des Textes aufgeschriebene rhetorische Frage: „Wohin geht ein Mensch, wenn er nicht weiß, wo er hingehen sollte“ (Erpenbeck 293). Als signifikant anklagend erscheint in diesem Zusammenhang eine der letzten Szenen im Roman, in der einer der Flüchtlinge namens Rufu an Richards Biedermeiersekretär sitzend die deutschen Tempusformen übt und dabei auf folgende Idee kommt:

Rufu sitzt an Richards Biedermeiersekretär vor seinem aufgeschlagenen Heft und sagt: Ich gehen.

Richard schaut ihm über die Schulter und korrigiert: Das heißt: Ich gehe.

Rufu: Ich gehen.

Richard: Nein, ich gehe!

Rufu: Ich will die deutschen Verben zerbrechen.

Zerbrechen, sagt Richard, ist aber ein sehr schönes Verb. (Erpenbeck 336) 


\section{ZITIERTE WERKE}

Adelson, Leslie, A. "Touching Tales of Turks, Germans, and Jews: Cultural Alterity, Historical Narrative, and Literary Riddles for the 1990s." New German Critique, vol. 80, 2000, pp. 93-124.

Adorno, Theodor. Negative Dialektik. Suhrkamp, 2000.

Apel, Friedmar. "Wir wurden, werden, sind sichtbar." FAZ, 16 May 2015, www.faz.net/aktuell/ feuilleton/buecher/rezensionen/belletristik/gehen-ging-gegangen-von-jenny-erpenbeck-13 770081.html. Accessed 24 Aug. 2018.

Birrer, Sibyllie. "Gestrandet in der Warteschlaufe." Neue Zürcher Zeitung, 10 Dec. 2015, www. nzz.ch/feuilleton/buecher/gestrandet-in-der-warteschlaufe-1.18627304. Accessed 4 June 2018.

Bremerich, Stephanie: “Aus der sicheren Seite. Jenny Erpenbeck Gehen, ging, gegangen.” Kritisch-lesen.de, 14 March 2016, kritisch-lesen.de/rezension/auf-der-sicheren-seite. Accessed 23 Aug. 2018.

Buchzig, Dana. „Trifft ein Berliner Professor auf Flüchtlinge.“ Spiegel Online, 2 Sept. 2015, www.spiegel.de/kultur/literatur/gehen-ging-gegangen-von-jenny-erpenbeck-rezension-a1050518.html. Accessed 23 Aug. 2018.

Erpenbeck, Jenny. Gehen, ging, gegangen. Knaus, 2015.

Hermes, Stephan. "Grenzen der Repräsentation Zur Inszenierung afrikanisch-europäischer Begegnungen in Jenny Erpenbecks Roman Gehen, ging, gegangen.” Acta Germanica. Jahrbuch des Germanistenverbandes im Südlichen Afrika, vol. 44, issue 1, 2016, pp. 179-191.

Jakobson, Roman. „Randbemerkungen zur Prosa des Dichters Pasternak.“ Slawische Rundschau, vol. 7, 1935, pp. 357-374.

Kjaerstad, Jan, and Jon Fosse. „Metapher und Metonymie. Ein Briefwechsel”. Translated by A. Gundlach. Schreibheft, vol. 48, 1996, pp. 15-28.

Laermann, Klaus. “'Nach Auschwitz ein Gedicht zu schreiben, ist barbarisch.' Überlegungen zu einem Darstellungsverbot." Kunst und Literatur nach Auschwitz, edited by Manuel Köppen, Erich Schmidt, 1993, pp. 11-15.

Levy, Daniel, and Natan Szneider. Erinnerung im globalen Zeitalter. Der Holocaust. Aktual. Suhrkamp, 2006.

Lorey, Isabell. Die Regierung des Prekären. Turia + Kant, 2012.

Lühmann, Hannah. "Ein Roman als Crashkurs in Flüchtlingskrise.“ Die Welt, 29 Aug. 2015, www.welt.de/kultur/literarischewelt/article145830887/Ein-Roman-als-Crashkurs-inFluechtlings kunde.html. Accessed 24 Aug. 2018.

Magenau, Jörg. „Ein Stückchen Acker in Ghana.“ Süddeutsche Zeitung, 30 Aug. 2015, www. sueddeutsche.de/kultur/longlist-zum-deutschen-buchpreis-ein-stueckchen-acker-in-ghana1.2627330. Accessed 24 Aug. 2018.

Pokrywka, Rafał. "Drei Metaphern des Exils im neuesten deutschsprachigen Roman: Gehen, ging, gegangen von Jenny Erpenbeck, Ohrfeige von Abbas Khider, Das Mädchen mit dem Fingerhut von Michael Köhlmaeir.” Tematy i Konteksty, no. 7 (12), 2017, pp. 412-425.

Reinert, Bastian. “'Ich lasse am liebsten Tote sprechen.' Elfride Jelineks postmortales Theater.” Der Nationalsozialismus und die Shoah in der deutschsprachiger Gegenwartsliteratur, edited by Torben Fischer and Philipp Hammersteiner, Radoppi Verlag, 2014, pp. 259-273. 
Roth, Daniela H. Migration und Adoleszenz: Die (Un-)Möglichkeit transnationaler Handlungsfreiheit in Alina Bronskys Scherbenpark (2008), Steven Uhlys Adams Fuge (2011) und Martin Horváths Mohr im Hemd oder wie ich auszog, die Welt zu retten (2012), https://ub-madoc.bib.uni-mannheim.de/44302/1/Roth\%20Daniela_Die\%20Unm\%C3\% B6glichkeit\%20transnationaler\%20Handlungsfreiheit.pdf . Web. 4.06.2018.

Rothberg, Michael: "Von Gaza nach Warschau: Kartierung des multidirektionalen Gedächtnisses." Holocaust'-Fiktion. Kunst jenseits der Authentizität, edited by Iris Roebing-Grau and Dirk Rupnow. Wilhelm Fink, 2015, pp. 37-63.

Sarrazin, Thilo. Deutschland schafft sich ab. DVA, 2010.

Sternburg von, Judith. "Jedermann und die Afrikaner." Frankfurter Rundschau, 17 Sept. .2015, www.fr.de/kultur/literatur/jedermann-afrikaner-11693352.html. Accessed 24 Aug. 2018.

Trojanow, Ilija. Nach der Flucht. S. Fischer, 2017.

Wiesel, Elie. "The Holocaust as Literary Inspiration." Dimensions of the Holocaust, edited by Elie Wiesel, Lucy Dawidowicz, Dorothy Rabinovitz, and Robert McAfee Brown, Northwestern University Press, 1999, pp. 5-19.

\section{POSTFIGURACJE SZOAH WE WSPÓŁCZESNYM DYSKURSIE UCHODŹCZYM W POWIEŚCI JENNY ERPENBECK GEHEN, GING, GEGANGEN (2015)}

$$
\text { Streszczenie }
$$

Powieść Jenny Erpenbeck Gehen, ging, gegangen, która poświęcona jest współczesnej migracji, ukazuje poprzez zmieniającą się konstrukcję głównego bohatera oraz jego zaangażowanie w pomoc uchodźcom komplementarną relację między narracją o Szoah a aktualnym dyskursem migracyjnym w Europie. Emerytowany profesor klasyki poszukuje w obliczu nieprzestrzegania praw człowieka wobec współczesnych uchodźców narracyjnego ratunku w metonimicznych odniesieniach do kolonializmu i Szoah. Paradoksem zastosowania transfiguracji Zagłady jest w tym kontekście fakt, że niewyrażalność doświadczenia Szoah służy literackiej krytyce i obnażeniu degeneracji europejskiego prawodawstwa, u którego podstaw leży zasadzająca się na pamięci Holocaustu światowa Deklaracja Praw Człowieka z 1948 r.

Słowa kluczowe: powieść migracyjna; transfiguracja; Szoah.

\section{POSTFIGURATIONS OF THE SHOAH IN CONTEMPORARY REFUGEE DISCOURSE IN NOVEL BY JENNY ERPENBECK GEHEN, GING, GEGANGEN (2015)}

\section{S u m m ary}

Jenny Erpenbeck's refugee novel Gehen, ging, gegangen [Go, Went, Gone] explores the fundamental complementarity of the Shoah narrative and the current asylum and refugee discourse in Europe, through the spiritually ever-changing protagonist and his encounter with refugees in contemporary Germany. In the face of the decay of human rights, this retired professor of classical philology seeks "refuge" in references to the Shoah and colonialism. The paradox of this transfiguration consists of the fact that the ineffability of the genocide of the Jews in this novel serves to illuminate the social present and to denounce the perversion of the European legislation, which has its roots as the decisive spark for modern human rights in the aftermath of the Shoah itself.

Key words: migration novel; transfiguration; Shoah. 